\title{
Hydromechanics of swimming propulsion. Part 3. Swimming and optimum movements of slender fish with side fins
}

\author{
By T. YAO-TSU WU \\ California Institute of Technology, \\ Pasadena, California
}

(Received 21 July 1970)

This paper seeks to evaluate the swimming flow around a typical slender fish whose transverse cross-section to the rear of its maximum span section is of a lenticular shape with pointed edges, such as those of spiny fins, so that these side edges are sharp trailing edges, from which an oscillating vortex sheet is shed to trail the body in swimming. The additional feature of shedding of vortex sheet makes this problem a moderate generalization of the paper on the swimming of slender fish treated by Lighthill (1960a). It is found here that the propulsive thrust depends not only on the virtual mass of the tail-end section, but also on an integral effect of variations of the virtual mass along the entire body segment containing the trailing side edges, and that this latter effect can greatly enhance the thrust-making.

The optimum shape problem considered here is to determine the transverse oscillatory movements a slender fish can make which will produce a prescribed thrust, so as to overcome the frictional drag, at the expense of the minimum work done in maintaining the motion. The solution is for the fish to send a wave down its body at a phase velocity $c$ somewhat greater than the desired swimming speed $U$, with an amplitude nearly uniform from the maximum span section to the tail. Both the ratio $U / c$ and the optimum efficiency are found to depend upon two parameters: the reduced wave frequency and a 'proportionalloading parameter', the latter being proportional to the thrust coefficient and to the inverse square of the wave amplitude. The basic mechanism of swimming is examined in the light of the principle of action and reaction by studying the vortex wake generated by the optimum movement.

\section{Introduction}

Lighthill (1960a) investigated the inviscid flow around a slender fish which makes swimming movements in a direction transverse to its direction of locomotion, while its cross-section varies along it only gradually. Based on the slender-body theory, Lighthill obtained the result of thrust produced by the fish, time-rate of work done by it, and the rate of shedding of energy, showing that the mean values of these quantities all depend on the movement and body shape at the tail-end section only, and that they will vanish with the virtual mass of the tail. What has primarily been implied here is that the body cross- 
section varies so gradually, and its shape is so smooth (no sharp edges), that the cross-flow remains attached to body, leaving no vortex sheet until the tailend section is reached. This situation may represent, quite accurately, several wide classes of aquatic animals, such as the eel Anguilla vulgaris, eel-like fishes of the order Heteromi, ribbon fishes of the order Allotriognathi, members of the order Anacanthini like cod and similar fishes, as have been discussed in an interesting review article by Lighthill (1969).

There exist, however, other classes of fishes of more advanced orders which are known as strong, active swimmers, capable of putting on impressive performances. This group of fish orders contains members of the order Isospondyli (such as salmon, trout, etc.) and those of the large order Ostariophysi, to which belong most of the successful freshwater fishes. As an indicative measure of the over-all performance, the swimming speed $U$ is usually expressed by biologists in units of body-length/sec, or $n=U / l$. For instance, pike (Esox lucius), salmon (Salmo salar), trout (Salmo gairdneri), dace (Leuciscus leuciscus), and herring (Clupea harengus) have been observed to range from $n=6 /$ sec to $12 / \mathrm{sec}$ (see Hertel 1963, chap. G; Gray 1968, chap. 3). Speeds considerably greater than 10/sec, as high as 20/sec, have also been recorded by Walters \& Fiersteine (1964) for tunny (Thunnus albacores) and wahoo in open water. By and large, the slenderness parameter, $\delta$, defined as the ratio of maximum depth (or height, or called span as in the wing theory) to body length, of this general group of fishes is moderate to small (about from 0.4 down to 0.18 based on body depth including dorsal and ventral fins extended, the smallest $\delta$ being represented by the fastswimming Pacific saury, Cololabis saira). Their thickness in the third dimension is yet smaller than their span; the ratio of minor to major axis of cross-section of some slightly compressed species may be as much as $0 \cdot 6$ all the way back to the caudal peduncle (point of minimum depth). As a general feature, the transverse cross-sections of these fishes have rather rounded edges anterior to the section of maximum depth, turning to a more or less lenticular shape with fairly pointed edges to the rear part of body in which may be found a great variety of dorsal, ventral, pectoral, anal, and possibly other smaller fins, to be followed by the caudal fin.

As for the detailed fin shapes and locations, there are perhaps as many different configurations as the number of species. However, as a crude classification, the dorsal and ventral fins may be arranged for hydromechanical reasons into two main types: the elongated 'ribbon fin' and the triangular or trapezoidal 'sailshaped fin'. Some pre-eminent families of fast fishes equipped with conspicuous ribbon-fins are dolphin fish Coryphaena hippurus, which has a dorsal ribbon-fin all the way from head to caudal peduncle and a shorter ventro-anal one, yellowtail Seriola quinqueradiata, atka mackerel Pleurogrammus azonus, porgies and breams (e.g. Pagrus major, Dentex tumifrons). Examples of fast fishes having sail-shaped dorsal and ventral fins are mullet Mugil cephalus, which is very fast and migrates widely, barracuda Sphyraena pinguis, trout, salmon, and herring. Also, many families of fishes have both types of fins, notably bluefin tuna Thunnus thynnus, skipjack, and some mackerels. Furthermore, several fishes (wahoo, tuna, skipjack, mackerel and saury) have behind the main fins a series 
of small fins, protruding with alternate angles of yaw, which are probably for boundary-layer control.

In order to simplify the mathematical analysis, we shall limit our consideration here to the ribbon-fin-type problem, assuming that the trailing side-edges have a gradual change in slope, but are sharp enough to shed an oscillating vortex sheet from the body in an undulatory swimming motion. The main purpose of this study is to adopt this relatively simple ribbon-fin form to determine the effect of vortex-sheet shedding on the swimming performance and to evaluate the optimum shape of movements which will give the maximum hydrodynamic efficiency under the influence of the vortex sheet. In this respect the present study was originally meant as a moderate generalization of the earlier work of Lighthill (1960a). However, at its completion, Lighthill (1970) became known to the author, in which the effect of vortex sheets shed from dorsal and ventral fins with straight trailing edges has been discussed from a somewhat different, but interesting, view-point. It is assumed, however, that the body sections behind the dorsal and ventral fins are smoothly shaped and do not shed any further vorticity. This is reasonable for many fishes including the freshwater Ostariophysi, such as dace (see Bainbridge 1963). The case considered by Lighthill is therefore only slightly different from the one presented here. His solution has made possible some comparative discussions in the later passages, and has probably placed an all-embracing theory within reach.

In applying the slender-body theory (for a general review see Lighthill 1960b) to the present problem, it appears that the case of unsteady flows past a slender body having trailing side-eges, from which an oscillating vortex sheet is shed, has not yet been developed (except, of course, by Lighthill 1970). This gap of development may perhaps be attributed to the general understanding that the trailing side-edges present no problem in steady slender-body flows, since the rear part of a slender wing aft the maximum span section carries no lift. This assertion, however, is no longer true for unsteady flows. Since the vorticity, after being shed from a trailing side-edge, is propagated downstream with the local velocity, its value at a station in the vortex wake depends on its strength at the trailing edge when it was shed at a retarded time proper to the distance apart. Consequently, unlike in the steady flow case, the unsteady cross-flow field, at least the velocities, cannot be determined for a local section without giving regard to the preceding sections. It may be said that, in the presence of an oscillating vortex wake, the flow is no longer 'slender', in the usual aerodynamical sense of the word, even though the body shape is still slender.

In this paper, the difficulty arising from the influence of upstream stations upon the vortex wake is effectively curtailed by working conjointly with the velocity and Prandtl's acceleration potential, as presented in $\$ 2$. The problem of optimum shape of swimming movements employed by slender fish is investigated in $\S 5$ by applying the method of variational calculus. The basic mechanism of swimming is examined from a different point of view based on the principle of action and reaction by evaluating the vortex wake generated by the optimum movement. Miscellaneous effects of finite body thickness, geometrical asymmetry, and of variable forward speed of swimming are discussed in the appendix. 


\section{The unsteady slender-body theory}

We consider the swimming motion of a slender fish, or rather a fish-like model, performing a time-harmonic transverse motion of small amplitude, while maintaining a constant rectilinear forward velocity $U$ through an incompressible fluid, which is otherwise at rest. (This theory can readily be extended to the general case of time-dependent rectilinear velocity $U(t)$. For the details see appendix, $\S($ iii).) The Reynolds number $R=U l / \nu$ based on the body length $l$ and kinematic viscosity $v$ of the fluid is taken to be large so that the boundary layer will be thin, and the inertial effects can be evaluated by the potential theory. The typical shape of the 'fish-like' body is characterized (see figure 1) by its slenderness in the spanwise direction compared with the length $l$ and its small thickness in the other transverse direction, as already noted in $\S 1$. Its transverse cross-sections are thin, having rather rounded edges anterior to the widest span section, turning to a lenticular shape with fairly pointed side edges to the hind part of body, followed by a tail-base neck just ahead of the caudal fin. The leading edges are assumed to be sufficiently round to prevent flow separation. The Kutta condition is assumed to hold valid at sharp trailing edges, from which an oscillating vortex sheet is shed to trail the body in unsteady motion. It is further assumed that the transverse displacement of the vortex sheet is small, the roll-up of the tip vortices not significant. Intuitively speaking, the degree or extent by which these assumptions are satisfied will have a more pronounced influence on the validity of the theory for slender bodies than for bodies of large aspect ratio since the shed vortex sheet will traverse for a longer duration alongside a slender body than a short one, before eventually leaving its rear end. Violation of these simplifying assumptions will give rise to the non-linear effects. Finally, we note that the effect of small thickness of a planar, slender body as specified here can be regarded as secondary. The body will therefore be taken in the sequel to be of zero thickness in order to simplify the analysis. The effect of finite body thickness is easily accounted for, as will be discussed separately in the appendix, §(ii).

It may be remarked here, at least for background information, that the actual situation of various categories of fishes in swimming is perhaps more complicated. A primary aspect is concerned with the generation of vortices and their penetration into the interior of fluid. Using a small fish (Brachydanio albolineatus) about $1.5 \mathrm{in}$. in length and swimming at about $24 \mathrm{in}$. $\mathrm{sec}^{-1}$, Rosen (1959) observed that the vortices originate just behind the gills and, having formed, pass backwards, swinging from one side to the other of the body without breaking the vortex lines, while their strength increases along the body. Another important aspect is in regard to the presence of higher harmonics in the periodic motion. In studying the movements of individual segments of fish body (including the glass-eel, Anguilla), Gray (1968) found that there appears greater deviation from the sinusoidal mode, the farther back the segment to the posterior end, thus indicating the presence of higher harmonies. As for the detailed movement of the more flexible parts of body, such as caudal fins, Bainbridge (1963) demonstrated that, during each transverse sweep, the caudal fin of such 
a fish as a dace (Leuciscus leuciscus) exhibits two modes of bending (dorsoventral and antero-posterior), and these changes appear to be under the control of the animal. Although the problem may be further complicated by these refined features, it is thought that the present formulation and its theoretical solution will be accurate enough to describe and predict the principal mechanism of swimming of slender fish. Some of these added features, such as the effect of the higher harmonics, can still be evaluated within the framework of the present theory.
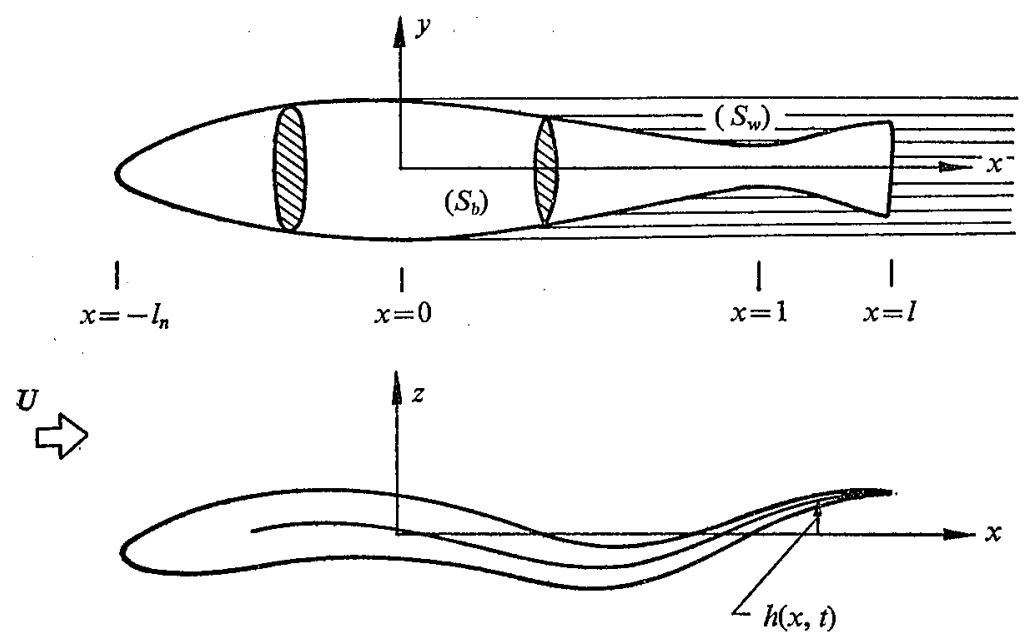

FIgURE 1. The co-ordinate system in the body frame of reference.

We choose a Cartesian co-ordinate system $(x, y, z)$ fixed at the mean position of the body, which is assumed to be of zero thickness, with the stretched body plan form $S_{b}$ lying in the $z=0$ plane and with the $x$-axis pointing in the stream direction. The projection of the trailing vortex sheet onto the $z=0$ plane will be denoted by $S_{w}$, which will be called the vortex-wake plan form. The complementary part of the $z=0$ plane outside $S_{b}+S_{w}$ will be denoted by $S_{c}$. The body motion can be written as

$$
z=h(x, t) \quad\left(x, y \in S_{b}\right),
$$

which is assumed for simplicity to be independent of $y$ but can be extended to the more general case with the $y$-dependence. The velocity $\mathbf{q}$ produced by this body motion in the free stream of velocity $U$ has a perturbation potential $\phi$,

$$
\mathbf{q}=(U+u, v, w)=\operatorname{grad}(U x+\phi)
$$

satisfying, on account of the continuity, the Laplace equation

$$
\nabla^{2} \phi=0 .
$$

The linearized Euler's equation of motion reads

where

$$
\begin{gathered}
D \mathbf{q}=\nabla \Phi, \quad \Phi=\left(p_{\infty}-p\right) / \rho \\
D \equiv \frac{\partial}{\partial t}+U \frac{\partial}{\partial x}
\end{gathered}
$$


being the linearized material differential operator, $\Phi$ is Prandtl's acceleration potential, $p$ the pressure, $p_{\infty}$ its value at infinity, and $\rho$ the density of the fluid. From (2) and (4) it follows, upon integration from $x=-\infty$, that

$$
D \phi=\Phi \text {. }
$$

Whence, by (3), $\Phi$ also satisfies the Laplace equation

$$
\nabla^{2} \Phi=0 .
$$

The boundary conditions of this problem, in terms of either $\phi$ or $\Phi$, are

$$
\begin{aligned}
\phi_{z}^{ \pm}=D h \equiv V(x, t), & \text { or } & \Phi_{z}^{ \pm}=D V & \left(x, y \in S_{b}\right), \\
D \phi^{ \pm}=0, & \text { or } & \Phi^{ \pm}=0 & \left(x, y \in S_{w}\right), \\
D \phi^{ \pm}=0, & \text { or } & \Phi^{ \pm}=0 & \text { (at trailing edges), } \\
\phi^{ \pm}=0, & \text { or } & \Phi^{ \pm}=0 & \left(x, y \in S_{c}\right), \\
\phi \rightarrow 0, & \text { or } & \Phi \rightarrow 0 & \text { (as } \left.x^{2}+y^{2}+z^{2} \rightarrow \infty\right) .
\end{aligned}
$$

Here the notation $\phi_{z}^{ \pm}$stands for the value of $\partial \phi / \partial z$ as $z \rightarrow \pm 0$, etc. Condition (8a) for $\Phi \pm$ follows from (7), which implies that $\Phi$ is odd in $z$, and from the requirement that the pressure $p$ must be continuous across the vortex sheet. Condition ( $8 b)$ is the Kutta condition, which we accept as valid. Condition (9) for $\phi^{ \pm}$results from integration of $D \phi \pm=0$ from $x=-\infty$ under condition (10). Condition (10) for $\phi$ excludes the region $z=0$, and $x, y$ on $S_{w}$.

The above formulation has been expressed in terms of either the velocity potential $\phi$ or the acceleration potential $\Phi$. The problem in terms of $\Phi$ is seen to be of a mixed-type boundary problem. When working with $\phi$, however, we note from condition (8) that the vorticity strength of the vortex sheet is a function of $(x-U t)$, and hence depends on its value at the trailing edge, evaluated at a retarded time. This latter value is, however, not known in advance, thus making the solution in terms of $\phi$ somewhat more complicated.

We shall next introduce the slender-body approximation which will help simplify the analysis and will yield a solution valid near the body. The body configuration will be assumed to be symmetrical with respect to $y$, so that the side edges are given by $y= \pm b(x)$. This assumption is mainly for simplicity and is not essential, since the more general case of asymmetrical shape, in $y$, can be dealt with with minor modifications (for the details see appendix $\S(\mathbf{i})$ ). The origin is taken to be at the mid-point of the maximum span so that $y=b(0)=b_{0}$ is the maximum of $b(x)$, and that the body, when stretched straight, extends from its nose at $x=-l_{n}$ to its tail end at $x=l$, the total length being $l_{T}=l_{n}+l$. Furthermore, the body may admit, just in front of the caudal fin, a tail-base neck at $x=l_{m}$, which will be normalized to be of unit length. (If the tail does not have a narrow neck, $l$ will be taken as the reference length, or $l=1$.) For slender bodies, the slenderness parameter is small,

$$
\delta=b_{0} / l_{T} \ll 1 \quad\left(l_{T} \equiv l_{n}+l\right) .
$$

Then, within a region near the body, (3) and (6) can be approximated, according to the slender-body theory (see Lighthill $1960 a, b$ ), by the two-dimensional Laplace equation

$$
\phi_{y y}+\phi_{z z}=0, \text { or } \Phi_{y y}+\Phi_{z z}=0
$$

with an error at most of order $O\left(\delta^{2}\right)$ relative to the terms retained. 
The two equations in (12) imply the existence of two conjugate harmonic functions, $\psi$ and $\Psi$, satisfying

$$
\phi_{y}=\psi_{z}, \quad \phi_{z}=-\psi_{y} ; \quad \Phi_{y}=\Psi_{z}, \quad \Phi_{z}=-\Psi_{y} .
$$

Consequently, in terms of the complex variable $\zeta=y+i z$,

$$
f=\phi+i \psi, \quad F=\Phi+i \Psi, \quad \Omega=d f / d \zeta=v-i w
$$

are all analytic functions of $\zeta$, regular everywhere within and on the flow boundary, save at a sharp leading edge and at the points of body surface where the slope is discontinuous. By analytic continuation of (5), $f$ and $F$ are related by

$$
F(\zeta ; x, t)=D f=\left(\frac{\partial}{\partial t}+U \frac{\partial}{\partial x}\right) f(\zeta ; x, t),
$$

which can be integrated along the characteristics $x-U t=$ const., giving

$$
\begin{gathered}
f(\zeta ; x, t)=f\left(\zeta ; x_{0}, \tau\left(x_{0} ; x, t\right)\right)+\frac{1}{U} \int_{x_{0}}^{x} F\left(\zeta ; x_{1}, \tau\left(x_{1} ; x, t\right)\right) d x_{1}, \\
\tau\left(x_{1} ; x, t\right) \equiv \tau\left(x_{1}\right)=t+\left(x_{1}-x\right) / U .
\end{gathered}
$$

$\tau\left(x_{1} ; x, t\right)$, which will often be abbreviated as $\tau\left(x_{1}\right)$, is the retarded time for a signal to propagate with wave velocity $U$ from $x_{1}$ to arrive at $x$ at time $t$.

We proceed to determine the solution $F$, which is required to possess a correct singular behaviour at the leading edges, and to be regular at the trailing edges, by virtue of the Kutta condition. Perhaps the simplest way is to work with the complex velocity $\Omega$ in the front sections with leading side edges $\left(-l_{n}<x<0\right)$, and to start with the complex acceleration potential $F$ in the rear sections $(0<x<l)$ in which the trailing vortex sheet exists. Thus, the boundary conditions for the cross-flow can be written:

$$
\begin{array}{ccl}
(-b(x)<y<b(x)) & (|y|>b(x)) \\
w^{ \pm}=-\operatorname{Im}\left(\frac{d f}{d \zeta}\right)^{ \pm}=V(x, t) & v^{ \pm}=\operatorname{Re}\left(\frac{d f}{d \zeta}\right)^{ \pm}=0 & \left(-l_{n}<x<0\right), \\
-\operatorname{Im}\left(\frac{d F}{d \zeta}\right)^{ \pm}=D V(x, t) & \operatorname{Re}\left(\frac{d F^{\prime}}{d \zeta}\right)^{ \pm}=0 & (0<x<l),
\end{array}
$$

together with the conditions

$$
|d f| d \zeta \mid \rightarrow 0 \text { and }|d F / d \zeta| \rightarrow 0 \text { as }|\zeta| \rightarrow \infty
$$

The problems of $d f / d \zeta$ and $d F / d \zeta$ both turn out to be posed as a RiemannHilbert problem (see also figure 2), the general solution of which (e.g. when $V$ also depends on $y$ ) is known (see e.g. Muskhelishvili 1953). In the present case when $V$ is independent of $y$ and the body shape is symmetric in $y$, the solution is particularly simple. In fact, the solution of $\Omega=v-i w$ under condition (17), (19) is by inspection (or can be found to be in e.g. Lamb 1932, p. 85)

$$
\Omega=\frac{d f}{d \zeta}=i V(x, t)\left\{\zeta\left[\zeta^{2}-b^{2}(x)\right]^{-\frac{1}{2}}-1\right\} \quad\left(-l_{n}<x<0\right),
$$


from which it follows immediately

$$
\begin{aligned}
& f=\phi+i \psi=i V\left[\left(\zeta^{2}-b^{2}\right)^{\frac{1}{2}}-\zeta\right] \quad\left(-l_{n}<x<0\right), \\
& F=D f=D\left\{i V\left[\left(\zeta^{2}-b^{2}\right)^{\frac{x}{2}}-\zeta\right]\right\} \quad\left(-l_{n}<x<0\right),
\end{aligned}
$$

or, carrying out the differentiation,

$$
F=i(D V)\left[\left(\zeta^{2}-b^{2}\right)^{\frac{1}{2}}-\zeta\right]-i V b(D b)\left(\zeta^{2}-b^{2}\right)^{-\frac{1}{2}} \quad\left(-l_{n}<x<0\right) .
$$

The last term of (22b) shows that the pressure has a square-root singularity at the leading edges. In the above, the function $\left(\zeta^{2}-b^{2}\right)^{\frac{1}{2}}$ is defined in the $\zeta$-plane cut along the real $\zeta$-axis from $\zeta=-b$ to $b$ such that it tends to $\zeta$ as $|\zeta| \rightarrow \infty$.

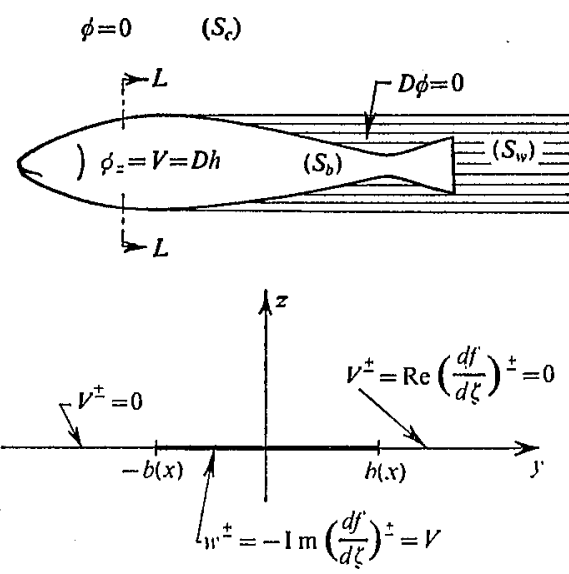

Section $L-L\left(-I_{n}<x<0\right)$

$$
\Phi=0 \quad\left(S_{c}\right)
$$
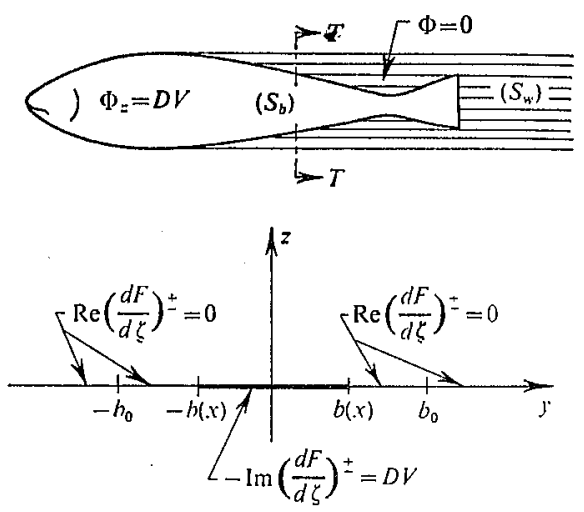

Section $T-T(0<x<h)$

Figure 2. The boundary problems of the front and rear sections of a slender body.

For $0<x<1$, or in the range with trailing side edges, we first note the complete analogy between the two sets of boundary conditions (17) for $d f / d \zeta$ and (18) for $d F / d \zeta$. Therefore, in analogy with the solution (21) of $f$, the solution of $F$ must be of the form

$$
F^{\prime}=(i D V)\left[\left(\zeta^{2}-b^{2}\right)^{\frac{1}{2}}-\zeta\right]+i C\left(\zeta^{2}-b^{2}\right)^{-\frac{1}{2}},
$$

in which the last term, with $C(x, t)$ being real, represents the only possible complementary solution which will yield a pressure even in $y$, integrable at the side edges and at infinity, and regular elsewhere. But $C=0$ by the Kutta condition $(8 b)$, and hence

$$
F=(i D V)\left[\left(\zeta^{2}-b^{2}\right)^{\frac{1}{2}}-\zeta\right] \quad(0<x<1),
$$

which is bounded everywhere within and on the flow boundary, and falls off at infinity. Now, a comparison between (24) and (22) shows that $F$, and hence also the pressure, will be continuous at the maximum span section if

$$
d b / d x \rightarrow 0 \text { as } x \rightarrow-0 \text { from the front side. }
$$

We shall assume this to be the case. (Actually, not satisfying condition (25) may be regarded as a weak violation of the slender flow approximation, since the local 
flow must then adjust itself to a rather steep longitudinal pressure gradient, such as the one at the transverse edge of a slender delta wing.)

The velocity potential in the range $0<x<1$ can be deduced by substituting (24) in (16). Writing $F$ in (24) first as

$$
F(\zeta ; x, t)=D\left\{i V\left[\left(\zeta^{2}-b^{2}\right)^{\frac{1}{2}}-\zeta\right]\right\}-i V U \frac{\partial}{\partial x}\left(\zeta^{2}-b^{2}\right)^{\frac{1}{2}},
$$

in which the operator $D$ in the last term, when applied to a function of $x$ only, has been reduced to $U \partial / \partial x$, we obtain, for $0<x<1$,

$$
\begin{aligned}
f(\zeta ; x, t) & =i V(x, t)\left[\left(\zeta^{2}-b^{2}\right)^{\frac{1}{2}}-\zeta\right]-i \int_{0}^{x} V\left(x_{1}, \tau_{1}\right) \frac{\partial}{\partial x_{1}}\left(\zeta^{2}-b_{1}^{2}\right)^{\frac{1}{2}} d x_{1} \\
& =i V\left(0, \tau_{0}\right)\left(\zeta^{2}-b_{0}^{2}\right)^{\frac{1}{2}}-i V(x, t) \zeta+i \int_{0}^{x}\left(\zeta^{2}-b_{1}^{2}\right)^{\frac{1}{2}} \frac{\partial V\left(x_{1}, \tau_{1}\right)}{\partial x_{1}} d x_{1},
\end{aligned}
$$

in which we have used the abbreviations

$$
\tau_{0}=\tau(0 ; x, t), \quad \tau_{1}=\tau\left(x_{1} ; x, t\right), \quad b_{0}=b(0), \quad b_{1}=b\left(x_{1}\right),
$$

where $\tau\left(x_{1}\right)$ is the retarded time given by $(16 b)$. The complex velocity is given by $v-i w=d f / d \zeta$, which is easily obtained from (26) and will not be reproduced here. We note that in the range $0<x<1, f$ and $\Omega$ are continuous everywhere at the plate, wherever $V$ is continuous, and also at the neighbouring vortex sheet, their derivatives being discontinuous only along the trailing edges. Furthermore, $f$ and $\Omega$ are also continuous across the maximum span section at $x=0$. However, $v$ and $w$ both have a square-root singularity just outside of the tip vortices at $y= \pm b_{0}$.

In the tail section $b<x<l$, in which the side edges are leading edges, the pressure may admit again a square-root singularity at the side edges, as in the front section. Since $\Phi=0$ at the vortex sheet, the presence of the vortex sheet alongside the tail section obviously will have no effect on the pressure distribution at the tail plate. Therefore, $F$ is given again by (22). The complex velocity potential $f$, however, no longer has the solution (21), but is given by the general integral of $(22 a)$, or

$$
f(\zeta ; x, t)=i V(x, t)\left[\left(\zeta^{2}-b^{2}\right)^{\frac{1}{2}}-\zeta\right]+g(\zeta ; x-U t),
$$

where $g$ is an arbitrary function of $\zeta$ and $(x-U t)$ so that $D g=0$. By requiring $f(\zeta ; x, t)$ to be continuous at $x=1$, using the above solution and $(26), g$ is readily determined, yielding, for $\mathbf{1}<x<l$,

$$
f(\zeta ; x, t)=i V(x, t)\left[\left(\zeta^{2}-b^{2}\right)^{\frac{1}{2}}-\zeta\right]-i \int_{0}^{1} V\left(x_{1}, \tau_{1}\right) \frac{\partial}{\partial x_{1}}\left[\zeta^{2}-b^{2}\left(x_{1}\right)\right]^{\frac{1}{2}} d x_{1} .
$$

The complex velocity can then be deduced from this $f$ by differentiation.

Finally, we note that the velocity potential $\phi$ is given by the real part of $f$ in these different regions and the longitudinal velocity component $u$ is simply (see (2)) $u=\partial \phi / \partial x$. 


\section{Circulation and vorticity}

We define the circulation around a contour $\mathscr{C}$, which starts from a point $(x, y, 0+)$ and ends up at $(x, y, 0-)$ without crossing the body or the wake plan form $\left(S_{b}\right.$ or $\left.S_{w}\right)$, by

$$
\Gamma(x, y, t)=\oint_{\mathscr{C}} \mathbf{q} \cdot d \mathbf{x}=\oint_{\mathscr{C}} \boldsymbol{\nabla} \phi \cdot d \mathbf{x}=\phi^{-}-\phi^{+} .
$$

Obviously, $\Gamma \neq 0$ only when $(x, y)$ falls within $S_{b}$ or $S_{w}$ since $\phi$ is discontinuous nowhere else. As $\phi$ is odd in $z$,

$$
\Gamma(x, y, t)=-2 \phi^{+}(x, y, t) \quad\left(x, y \in S_{b}+S_{w}\right) .
$$

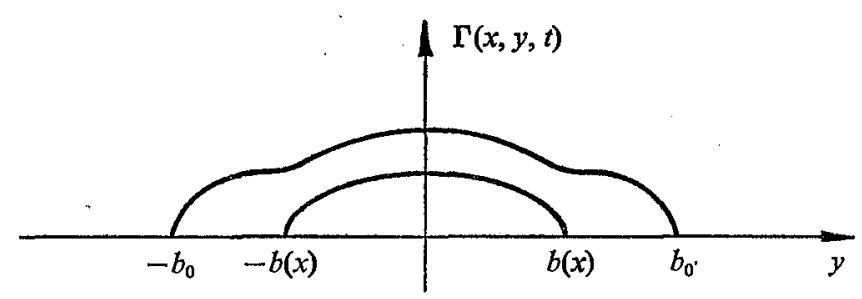

Frgure 3. The spanwise circulation distribution; the lower curve depicts $\Gamma$ in the front section, the upper one for a rear section.

From the previous solution of $f,(21),(26)$ and (28), we deduce the following:

(i) In the front section $\left(-l_{n}<x<0,|y|<b(x)\right)$, 。

$$
\Gamma(x, y, t)=2 V(x, t)\left[b^{2}(x)-y^{2}\right]^{\frac{1}{2}} .
$$

(ii) In the trailing-edge section $\left(0<x<1,|y|<b_{0}\right)$,

$$
\Gamma=2 V\left(0, \tau_{0}\right)\left(b_{0}^{2}-y^{2}\right)^{\frac{1}{2}}+2 \int_{0}^{x_{*}}\left(b_{1}^{2}-y^{2}\right)^{\frac{1}{2}} \frac{\partial V\left(x_{1}, \tau_{1}\right)}{\partial x_{1}} d x_{1},
$$

where

$$
\begin{aligned}
x_{*} & =b^{-1}(y) & & \left(b(x)<|y|<b_{0}, \quad 0<x<1\right) \\
& =x & & (|y|<b(x), \quad 0<x<1),
\end{aligned}
$$

$x=b^{-1}(y)$ being the inverse function of $y=b(x)$ for $0<x<1$.

(iii) In the tail section $\left(1<x<l,|y|<b_{0}\right)$,

$$
\Gamma=2 V(x, t)\left[b^{2}(x)-y^{2}\right]^{\frac{1}{2}} H(b-|y|)-2 \int_{0}^{x_{* *}} V\left(x_{1}, \tau_{1}\right) \frac{\partial}{\partial x_{1}}\left(b_{1}^{2}-y^{2}\right)^{\frac{1}{2}} d x_{1},
$$

where $H(b-|y|)=1$ for $|y|<b(x),=0$ otherwise; and

$$
\begin{aligned}
x_{* *} & =b^{-1}(y) & & \left(b(1)<|y|<b_{0}, \quad 0<x_{* *}<1\right) \\
& =1 & & (|y|<b(1)) .
\end{aligned}
$$

The circulation distribution $\Gamma(x, y, t)$ over $S_{b}$ and $S_{w}$ are continuous at the maximum span section $(x=0)$ and at the tail-base neck $(x=1)$. A qualitative picture of this $\Gamma$ distribution is shown in figure 3 .

The vorticity $\gamma$ of this flow is confined entirely to the body surface $S_{b}$ and the trailing vortex sheet $S_{w}$, and hence has only two components. Within the 
framework of the linearized theory, $\gamma=\left(\gamma_{1}, \gamma_{2}, 0\right)$. Since $\gamma$ is the curl of the velocity $\mathbf{q}, \operatorname{div} \gamma=0$, which becomes in this case

$$
\frac{\partial \gamma_{1}}{\partial x}+\frac{\partial \gamma_{2}}{\partial y}=0
$$

According to Helmholtz's theorem, the vorticity $\gamma_{1} \delta y$ distributed over a stretch of length $\delta y$ at $(x, y, 0)$ is equal to the circulation around the line element $\delta y$ in the positive sense (passing through the points $(x, y, 0-),(x, y+\delta y, 0-)$, $(x, y+\delta y, 0+),(x, y, 0+)$, in that order, see figure 4$)$, or

$$
\begin{aligned}
& \gamma_{1}(x, y, t)=v^{-}-v^{+}=-2 v^{+}=-2 \partial \phi^{+} / \partial y . \\
& \gamma_{2}(x, y, t)=u^{+}-u^{-}=2 u^{+}=2 \partial \phi^{+} / \partial x \text {. }
\end{aligned}
$$
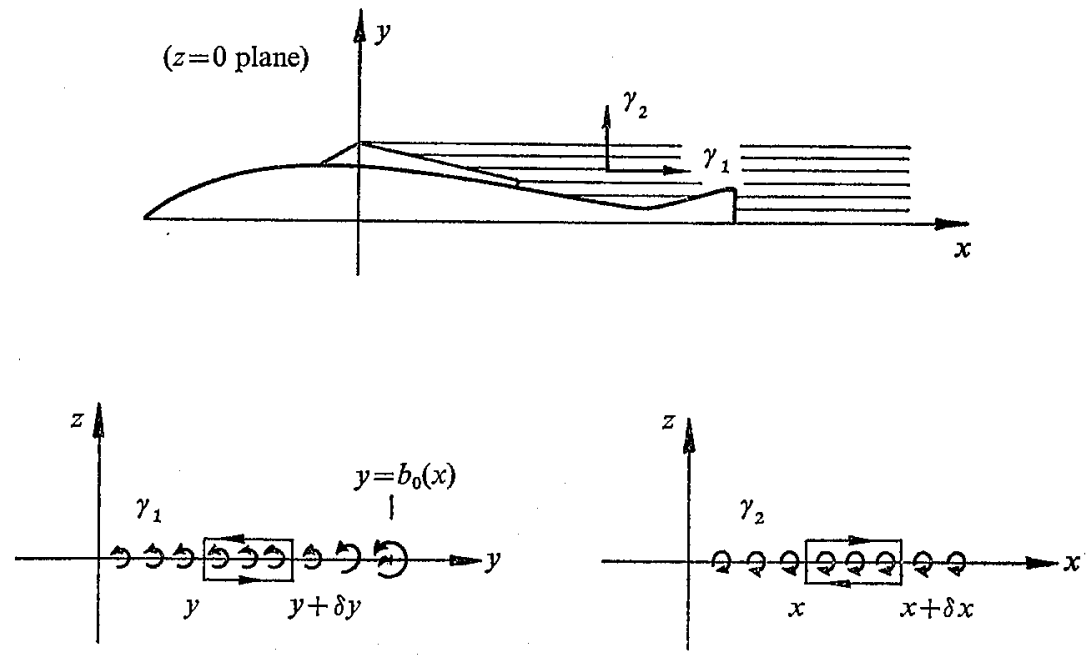

FiguRe 4. The vorticity components $\gamma_{1}, \gamma_{2}$.

Substitution of (34), (35) in (33) leads once again to the known statement of irrotationality of the flow outside the vortex sheet. From condition (8), $D \phi^{ \pm}=0$ on $S_{w}$, it follows that

$$
D \gamma_{1}=0 \quad \text { and } \quad D \gamma_{2}=0 \quad\left(x, y \in S_{w}\right)
$$

Now, substituting (29) in (34), we obtain

$$
\gamma_{1}(x, y, t)=\partial \Gamma / \partial y \quad\left(x, y \in S_{b}+S_{w}\right),
$$

which shows that shedding of the vorticity component $\gamma_{1}$ is due to the spanwise variation of $\Gamma$, the same as in the steady lifting-line or lifting-surface theory. Furthermore, by making use of condition (8) and relationship (29) in (35),

$$
\begin{aligned}
\gamma_{2}(x, y, t) & =-\partial \Gamma / \partial x & & \left(x, y \in S_{b}+S_{w}\right) \\
& =U^{-1} \partial \Gamma / \partial t & & \left(x, y \in S_{w}\right) .
\end{aligned}
$$

This asserts that shedding of the vorticity component $\gamma_{2}$ is due to the time variation of $\Gamma$ at the trailing edge, a feature in common with the two-dimensional oscillating airfoil theory. 


\section{Hydrodynamic forces and energy balance}

The pressure difference $\Delta p$ across the flexible plate is

$$
\Delta p=p^{-}-p^{+}=\rho\left(\Phi^{+}-\Phi^{-}\right)=2 \rho \Phi^{+}(x, y, t) \quad\left(x, y \in S_{b}\right),
$$

where, by (22) and (24),

$$
\begin{aligned}
& \Phi^{+}=-D\left\{V(x, t)\left[b^{2}(x)-y^{2}\right]^{\frac{1}{2}}\right\} \quad\left(-l_{n}<x<0, \quad 1<x<l\right), \\
& =-[D V(x, t)]\left[b^{2}(x)-y^{2}\right]^{\frac{1}{2}} \quad(0<x<1) .
\end{aligned}
$$

The instantaneous lift (or the force in the $z$-direction) per unit length of fish is

$$
\begin{aligned}
& \mathscr{L}(x, t)=\int_{-b}^{b}(\Delta p) d y=-2 \rho D \int_{-b}^{b} V(x, t)\left(b^{2}-y^{2}\right)^{\frac{1}{2}} d y=-\rho D[V(x, t) A(x)] \\
& \left(-l_{n}<x<0, \quad 1<x<l\right), \\
& =-2 \rho(D V) \int_{-b}^{b}\left(b^{2}-y^{2}\right)^{\frac{1}{2}} d y=-\rho[D V(x, t)] A(x) \quad(0<x<1), \\
& \rho A(x)=\rho \pi b^{2}(x)
\end{aligned}
$$

is the 'virtual mass' of the planar body in a longitudinal segment of unit length for motions in the $z$-direction. This result also holds valid for slender bodies asymmetrical in $y$ (see appendix $\S(i)$ ). In sections where no trailing vortex sheet is shed, $\mathscr{L}(x, t)$ is equal and opposite to the rate of change of cross-flow momentum of the fluid passing station $x$ at time $t$. The effect of vortex shedding, as shown by $(41 b)$, is equivalent to leaving the longitudinal rate of variation of the 'virtual mass ' out of consideration in calculating the changes of the cross-flow momentum. This significance of the above result can be utilized to help evaluate or explain other flow quantities.

The total lift in the $z$-direction is given by the integral of $\mathscr{L}$,

$$
L(t)=-\rho \int_{-l_{n}}^{l} D[V(x, t) A(x)] d x+\rho U \int_{0}^{1} V(x, t) \frac{\partial A}{\partial x} d x .
$$

The moment of force about the origin (positive if nose turns in the $z$-direction) is

$$
M(t)=\rho \int_{-l_{n}}^{l} x D[V(x, t) A(x)] d x-\rho U \int_{0}^{1} x V(x, t) \frac{\partial A}{\partial x} d x .
$$

The time averages of $L$ and $M$ for a fish in periodic motion are of course zero.

The thrust produced by a fish making a displacement $h(x, t)$ is $(\mathrm{Wu} 1971 a)$

$$
T=\int_{-l_{n}}^{l} \mathscr{L}(x, t) \frac{\partial h}{\partial x} d x+T_{s}
$$

where $T_{s}$ is the thrust due to the leading-edge suction. To obtain this $T_{s}$, we first observe that the complex velocity $v-i w=d f / d \zeta$ near the leading edge $\zeta=b(x)$ has the asymptotic representation

$$
v-i w \simeq i V\left(\frac{1}{2} b\right)^{\frac{1}{2}}\left[(\zeta-b)^{-\frac{1}{2}}+O(1)\right] \text { as }|\zeta-b(x)| \rightarrow 0,
$$

for both $-l_{n}<x<0$ and $1<x<l$, as can be shown using (20) and (28). From (21) and (28) it can also be seen that $u=\partial \phi / \partial x$ near the leading edge is of order 
$O(\delta)$ compared with $|v-i w|$, and hence $u^{2}$ can be neglected relative to $\left(v^{2}+w^{2}\right)$ in calculating the pressure near the leading edge. The singular suction force $Y_{s}-i Z_{s}$ is therefore given by

$$
Y_{s}-i Z_{s}=\frac{i \rho}{2} \oint_{|\zeta-b|=e}(v-i w)^{2} d \zeta=\frac{1}{2} \pi b \rho V^{2},
$$

which acts normal to the side leading edge, lying in the body plane $S_{b}$, and directed outwards from the body. The total suction thrust is therefore

$$
T_{s}=2\left(\int_{-l_{n}}^{0}+\int_{1}^{l}\right) Y_{s} \frac{d b}{d x} d x=\left(\int_{-l_{n}}^{0}+\int_{1}^{l}\right) \frac{1}{2} \rho V^{2}(x, t) \frac{d A}{d x} d x
$$

Finally, the total thrust becomes

$$
\begin{aligned}
T & =-\rho\left(\int_{-l_{n}}^{0}+\int_{1}^{l}\right)\left[h_{x} D(V A)-\frac{1}{2} V^{2} \frac{d A}{d x}\right] d x-\rho \int_{0}^{1} h_{x}(D V) A d x \\
& =-\rho \int_{-l_{n}}^{l}\left[D\left(V A h_{x}\right)-\frac{\partial}{\partial x}\left(\frac{1}{2} V^{2} A\right)\right] d x-\rho \int_{0}^{1} V\left(\frac{1}{2} V-U h_{x}\right) A_{x} d x \\
& =-\rho \frac{\partial}{\partial t} \int_{-l_{n}}^{l} A V h_{x} d x+\left[\rho A V\left(\frac{1}{2} V-U h_{x}\right)\right]_{x=l}-\rho \int_{0}^{1} V\left(\frac{1}{2} V-U h_{x}\right) A_{x} d x,
\end{aligned}
$$

in which we have taken the virtual mass at the nose $A\left(-l_{n}\right)=0$. Therefore, the mean over a long time of the thrust produced by the fish is

$$
\bar{T}=\frac{1}{2} \rho A(l)\left[\overline{\left[h_{t}^{2}-U^{2} h_{x}^{2}\right.}\right]_{x=l}-\frac{1}{2} \rho \int_{0}^{1} \overline{\left(h_{t}^{2}-U^{2} h_{x}^{2}\right)} \frac{d A}{d x} d x .
$$

The first term on the right side of (47) is the contribution of the tail-end section to the thrust, a known result which is attributed to Lighthill (1960a, equation (7)). The second term represents the contribution due to shedding of vortices, and is a positive contribution wherever $\left(h_{t}-U h_{x}\right)$ and $V=\left(h_{t}+U h_{x}\right)$ are positively correlated, since $d A / d x<0$ in the range $0<x<1$.

We next determine the power, $P$, delivered by the fish in making displacement $h(x, t)$; this power is equal to the rate of work done by the body against the reaction of cross-flow, $-\mathscr{L}(x, t)$, in the direction of body transverse velocity $h_{t}$, or

$$
\begin{aligned}
P & =-\int_{-l_{n}}^{l} \mathscr{L}(x, t) h_{t} d x=\rho \int_{-l_{n}}^{l}\left[D\left(A V h_{t}\right)-A V V_{t}\right] d x-\rho U \int_{0}^{1} V h_{t} A_{x} d x \\
& =\rho \frac{\partial}{\partial t} \int_{-l_{l}}^{l} A V\left(h_{t}-\frac{1}{2} V\right) d x+\rho U\left(A V h_{t}\right)_{x=l}-\rho U \int_{0}^{1} V h_{t} A_{x} d x .
\end{aligned}
$$

From this we have the mean power required as

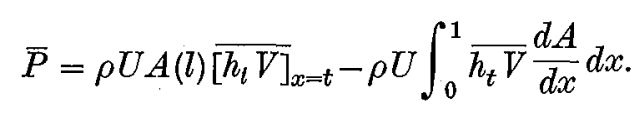

The first term is again Lighthill's result, which can be considered as the product of the lateral velocity $h_{t}$ of the tail with the rate of shedding of lateral momentum $(\rho A V) U$. It is also clear that the rate of shedding of lateral momentum from the trailing side edges between sections $A(x)$ and $A(x+d x)=A(x)+d A$ is 
$(-\rho V d A) U$ (the negative sign being taken since $d A=(d A / d x) d x<0$ for $0<x<1$ ), and that the product of this quantity with the local lateral velocity $h_{t}$ is $\left[-\rho U h_{t} V(d A / d x) d x\right]$. Integration of this product over the entire trailing side edges therefore yields the total contribution to power $P$ due to the effect of vortex shedding, which is the second term in (49).

To complete the energy balance, we consider next the kinetic energy $E$ imparted to the fluid in unit time,

$$
E=-\iint_{S_{b}}(p \mathbf{n}) \cdot \mathbf{q} d S=-\int_{-l_{n}}^{l} V(x, t) \mathscr{L}(x, t) d x-U T_{s},
$$

or, alternatively, we can apply the principle of energy conservation (Wu $1971 a$ ) that the power input $P$ must be equal to the rate of work done by the thrust, $T U$, plus the kinetic energy $E$ lost to the fluid in unit time,

$$
P=T U+E
$$

Therefore the mean energy loss is $\bar{E}=\bar{P}-U \bar{T}$, or

$$
\bar{E}=\frac{1}{2} \rho U A(l) \overline{V^{2}(l, t)}-\frac{1}{2} \rho U \int_{0}^{1} \overline{V^{2}(x, t)} \frac{d A}{d x} d x .
$$

Clearly, the rate of shedding of kinetic energy of cross-flow at the tail edge is $\left(\frac{1}{2} \rho A V^{2}\right)$ multiplied by $U$. The second term again represents the contribution due to shedding of vortices at the trailing side edges, the physical interpretation being entirely parallel to that for $\bar{P}$ given in the sequel to (49).

It is of importance to note from (52) that $\bar{E} \geqslant 0$, the equality holds if and only if $V(x, t) \equiv 0$ for $0<x<1$ and $x=l$, as should be expected on physical grounds (Wu 1971 $a$ ). From (51) and the result $\bar{E} \geqslant 0$, it follows

$$
\bar{P} \geqslant \bar{T} U \text {. }
$$

When the mean thrust is positive, the hydrodynamic efficiency, as defined previously, is

$$
\eta=U \bar{T} / \bar{P}
$$

This is a convenient place to compare the present theoretical result with the case treated by Lighthill (1970), which is for dorsal and ventral fins having straight trailing edges (so that the instantaneous strength of the vortex sheets shed from these fins is known a priori). It is further assumed by Lighthill that the body sections behind these fins are smoothly shaped, and do not shed any further vorticity. Under these circumstances the side force acting in the zdirection on the fish is given by (his equation (25), but converted to the present notation to aid comparison)

$$
\mathscr{L}=-\left(\frac{\partial}{\partial t}+U \frac{\partial}{\partial x}\right)[\rho A(x) V(x, t)]-\rho U \tilde{A}^{\prime}(x) V\left(x_{F}, t-\frac{x-x_{F}}{U}\right),
$$

where $x=x_{F}$ is where the vortex sheet starts and $\tilde{m}(x) \equiv \rho \tilde{A}(x)$ is the virtual mass associated with the vortex sheet of known strength in the presence of a completely stationary cylinder $C_{x}$ having the same cross-sectional shape as the body section at $x$. This result is slightly different from the side force $\mathscr{L}$ of $(41 b)$, 
essentially in that $V$ in the last term of the above equation assumes the retarded time whereas the corresponding $V$ in $(41 b)$ is evaluated at time $t$; this difference is the consequence of the different cases being considered. Lighthill's conclusions regarding thrust are accordingly slightly different from (46). In practice, however, these two theories come to much the same thing, because the extra term from the caudal-fin leading edge, which may modify the thrust (see the last term of Lighthill's equation (26)), has in effect only a small mean value owing to phase shifts.

\section{Harmonic motion: the optimum shape}

Of fundamental importance is the simple harmonic motion,

$$
h(x, t)=\operatorname{Re}\left[h_{1}(x) \exp (i \omega t)\right]=\operatorname{Re}\left[h_{1}^{*}(x) \exp (-i \omega t)\right]
$$

where $h^{*}$ stands for the complex conjugate of $h$. It is convenient to use the coefficient form of mean thrust, power required and energy loss as

$$
\begin{aligned}
C_{T} & \equiv \bar{T} /\left(\frac{1}{4} \rho U^{2} l_{m}^{2}\right)=Q_{T}(l) A(l)-\int_{0}^{1} Q_{T}(x) \frac{d A}{d x} d x, \\
C_{P} & \equiv \bar{P} /\left(\frac{1}{4} \rho U^{3} l_{m}^{2}\right)=Q_{P}(l) A(l)-\int_{0}^{1} Q_{P}(x) \frac{d A}{d x} d x, \\
C_{E} & \equiv \bar{E} /\left(\frac{1}{4} \rho U^{3} l_{m}^{2}\right)=Q_{E}(l) A(l)-\int_{0}^{1} Q_{E}(x) \frac{d A}{d x} d x,
\end{aligned}
$$

where $l_{m}=1$ is the unit length from the maximum span section to tail base,

being the reduced frequency, and

$$
\sigma=\omega l_{m} / U
$$

$$
\begin{aligned}
& Q_{T}(x)=\sigma^{2} h h^{*}-h_{x} h_{x}^{*}, \\
& Q_{P}(x)=i \sigma^{2} h h^{*}+i \sigma\left(h h_{x}^{*}-h^{*} h_{x}\right), \\
& Q_{E}(x)=Q_{P}-Q_{T}=\left(h_{x}+i \sigma h\right)\left(h_{x}^{*}-i \sigma h^{*}\right) .
\end{aligned}
$$

It is of interest to take note of the progressing transverse wave

$$
h=\xi_{0} \exp [-i(k x-\omega t)]=\xi_{0} \exp [-i k(x-c t)], \quad c=\omega / k,
$$

$\xi_{0}$ being a real constant. Corresponding to this body motion,

$$
C_{T}=\xi_{0}^{2}\left(\sigma^{2}-k^{2}\right)\left(A_{l}+A_{0}-A_{m}\right), \quad C_{P}=[2 \sigma /(\sigma+k)] C_{T}
$$

where

$$
A_{0}=A(0), \quad A_{m}=A\left(l_{m}\right), \quad A_{l}=A(l),
$$

and hence

$$
\eta=C_{T} / C_{P}=\frac{1}{2}\left(1+\frac{k}{\sigma}\right)=\frac{1}{2}\left(1+\frac{U}{c}\right) \text {. }
$$

First of all, we note that, with the effect of trailing vortex sheet taken into account, it is now possible to obtain propulsive thrust even if the tail has no structure, that is, with a pointed end such that $A_{l}=0$. Secondly, we see that the efficiency $\eta$ can become quite high when $(c-U) \ll U$, an important feature which has already been observed by Lighthill (1960a) and discussed for plane flows and other cases in part 2 (Wu 1971b) of this paper. In the limit as $c \rightarrow U$, 
or $k \rightarrow \sigma$, we obtain the special case in which $V=\left(h_{x}+i \sigma h\right) U \equiv 0$ and the corresponding $T, P$ and $E$ all vanish, whereas the efficiency $\eta$ tends to 1 . This special case has already been discussed in part 2 . It will be seen that, like the plane flow case, the optimum shape turns out to be a perturbation of this special case (see (73), in particular).

The optimum shape problem at hand can be stated as follows. Within the class of shape function $h$ of (55), required to be continuous in $x$ and to satisfy $|\partial h| \partial x \mid \ll 1$, find the optimum one which will minimize $C_{P}$ under the condition of fixed thrust coefficient

$$
C_{T}=C_{T, 0}>0 \text {. }
$$

Furthermore, it may be desirable to include the two recoil conditions (see part 1)

$$
\begin{gathered}
L(t)=\int_{-l_{s}}^{l} m(x) \frac{\partial^{2} h}{\partial t^{2}} d x, \\
M(t)=-\int_{-l_{h b}}^{l} x m(x) \frac{\partial^{2} h}{\partial t^{2}} d x,
\end{gathered}
$$

$m(x) d x$ being the mass of fish in the longitudinal segment $d x$ at $x$. We shall, however, consider here the optimum shape problem without enforcing the recoil conditions $(65),(66)$, this practice being acceptable since the $h$ in the front part $\left(-l_{n}<x<0\right)$ can be properly adjusted afterwards to satisfy (65) and (66).

The optimization under constraint (64) is equivalent to minimizing a new functional

where

$$
\begin{gathered}
I\left[h(x), h_{l}, \dot{h_{l}}\right]=\lambda C_{P}-\left(C_{T^{\prime}}-C_{T, 0}\right)=I_{1}\left(h_{l}, \dot{h}_{l}\right)+I_{2}[h(x)], \\
I_{1}\left(h_{l}, \dot{h_{l}}\right)=\left[\lambda Q_{P}(l)-Q_{T}(l)\right] A_{l}, \\
I_{2}[h]=-\int_{0}^{1} G(h, \dot{h}) \alpha(x) d x, \quad G(h, \dot{h})=\lambda Q_{P}(h, \dot{h})-Q_{T}(h, \dot{h}),
\end{gathered}
$$

and $\lambda$ is an undetermined multiplier. In the above, the dependence of the fundamental function $G$ on $h, h_{x}$ as well as their complex conjugate is understood. In the next step, when the shape function $h$ is given an arbitrary variation $\delta h(x)$ in $0<x<1$ and a variation at the tail $x=l$, it is of importance to realize that, in this extremization, the amplitude of $h$ at some point must be left free as a reference amplitude, since the theory, being a linearized one, will not be altered when the solution is uniformly magnified. Therefore, in the variation of $I_{1}, h_{l}$ may be held free or, in other words, the extremum of $I_{1} / h_{l}^{2}$ is to be calculated for an arbitrary variation of $\dot{h}_{l} / h_{l}$. For the same reason, the amplitudes $h_{0}=h(0)$ and $h_{m}=h\left(l_{m}\right)=h(1)$ will be left free in the variation of $I_{2}$. To fail to observe these free end conditions will lead again to the difficulties as discussed in part 2.

With the end conditions so clarified, we proceed to impose on $h$ an arbitrary variation $\delta \dot{h}_{l}$ at $x=l$ and a variation $\delta h(x)$ in $0<x<1$, yielding the corresponding first variations of $I_{1}$ and $I_{2}$ as

$$
\begin{aligned}
\delta I_{1} & =A_{l}\left[h_{x}+i \lambda \sigma h\right]_{x=l} \delta \dot{h}_{l}^{*}+\text { complex conjugate } \\
\delta I_{2} & =-\int_{0}^{1}\left[\left(\partial G / \partial h^{*}\right) \delta h^{*}+\left(\partial G / \partial \dot{h}^{*}\right) \delta \dot{h}^{*}\right] \alpha(x) d x+\text { complex conjugate } \\
& =-\left.\frac{\partial G}{\partial \dot{h}^{*}} \alpha(x) \delta h^{*}\right|_{x=0} ^{1}+\int_{0}^{1}\left\{\frac{d}{d x}\left[\alpha \frac{\partial G}{\partial \dot{h}^{*}}\right]-\frac{\partial G}{\partial h^{*}} \alpha\right\} \delta h^{*} d x+\text { c.c. }
\end{aligned}
$$


In order that $I\left[h(x), h_{l}, \dot{h}_{l}\right]$ be extremum, $\delta I_{1}$ and $\delta I_{2}$ must vanish separately for arbitrary $\delta \dot{h}_{l}$ and $\delta h(x)$. This yields from $(68 a)$ the end condition

$$
h_{x}+i \lambda \sigma h=0 \quad(x=l),
$$

and from $(68 b)$ the Euler-Lagrange equation

$$
\frac{d}{d x}\left[\left(h_{x}+i \lambda \sigma h\right) \alpha(x)\right]+\left[i \lambda \sigma h_{x}-(2 \lambda-1) \sigma^{2} h\right] \alpha(x)=0 \quad(0<x<1),
$$

and the transversality conditions $\dagger$

$$
\left(h_{x}+i \lambda \sigma h\right) \alpha(x)=0 \quad(x=0,1) .
$$

For slender bodies having a maximum span at $x=0$ and a minimum at $x=1$, it is typical that $\alpha(x)$ has simple zeros at $x=0,1$, and $\alpha<0$ for $0<x<1$. Then $(70 a)$ is an ordinary differential equation, $x=0,1$ being its two regular singularities. Now, by change of variable,

$$
h(x, t)=g(x) \exp [i(\omega t-\lambda \sigma x)],
$$

equation $(70 a)$ and the transversality condition $(70 b)$ become

$$
\begin{array}{rlrl}
{\left[\alpha g_{x}\right]_{x}+\mu \alpha g=0} & & \left(0 \leqslant x \leqslant 1, \quad \mu=(1-\lambda)^{2} \sigma^{2}\right), \\
\alpha(x) g_{x}=0 & & (x=0,1) . &
\end{array}
$$

This problem of $g(x)$ may have several possible typès. (i) $\alpha(x)$ is negative and does not vanish in $0 \leqslant x \leqslant 1$ (e.g. when $b^{\prime}(x)$ has a jump at $x=0,1$ ): then (72a,b) belong to the standard Sturm-Liouville system. (ii) $\alpha<0$ in $0<x<1$, $\alpha$ has simple zeros at $x=0,1$, and, by choice, $g_{x}=0$ at $x=0,1$ : this is a singular Sturm-Liouville problem. $\ddagger$ (iii) $\alpha(x)$ has the same properties as in (ii), and the boundary conditions merely require $g(x)$ to remain finite, so that $(72 b)$ is still satisfied: this case is no longer an eigenvalue problem. Case (i) does not have general interest, whereas, in case (ii), the eigenfunctions, $\ddagger$ except possibly for the lowest eigenvalue $\mu_{0}=0$, with corresponding eigenfunction $g_{0}(x)=$ const., are seen to bear no physical relevance with high efficiency.

Case (iii) is of general interest. To obtain an approximate solution for this case, we note that, at high efficiencies, $(1-\lambda)$ will be small, so will be $\mu$ if $\sigma$ is not too large. Consequently, up to a term of order $O(\mu \log \mu)$, the only regular solution of $(72 a)$ is $g_{0}(x)=$ const., which renders all three conditions in (69) and $(72 b)$ completely satisfied. Thus we have, as the first-order solution, the optimum shape as

$$
h(x, t)=\xi_{0} \exp [i(\omega t-\lambda \sigma x)] \quad\left(\xi_{0}=\text { const. }\right) .
$$

$\dagger$ These conditions must still be satisfied even when $\alpha(0) \neq 0, \alpha(1) \neq 0$.

$¥$ It can be shown for case (ii) that the eigenvalues of (72a), infinite in number, are non-negative, and form a discrete spectrum, and that the eigenfunctions are orthogonal to each other over $(0,1)$ weighted with respect to $\alpha(x)$. For instance, if $\alpha(x)=\sin \pi x$, then the eigenvalues are $\mu_{n}=n(n+1) \pi^{2}(n=0,1,2, \ldots)$, associated with the eigenfunctions $g_{0}=1, g_{1}=\cos \pi x, g_{2}=1+3 \cos 2 \pi x, g_{3}=3 \cos \pi x+5 \cos 3 \pi x$, etc. For further discussion of singular Sturm-Liouville problems, see Weyl (1910), Courant \& Hilbert (1953). 
The Lagrange multiplier $\lambda$ can now be determined by condition (64), yielding

$$
C_{T, 0}=\left(\xi_{0} \sigma\right)^{2}\left(1-\lambda^{2}\right)\left(A_{l}+A_{0}-A_{m}\right)
$$

which gives for $\lambda$ two real roots, $\lambda_{1}$ and $\lambda_{2}$,

where

$$
\begin{gathered}
\lambda_{1}=-\lambda_{2}=\left(1-\sigma_{c}^{2} / \sigma^{2}\right)^{\frac{1}{2}} \quad\left(\sigma \geqslant \sigma_{c}\right), \\
\sigma_{c}^{2}=\tilde{C}_{T, 0}=C_{T, 0} /\left(\xi_{0}^{2} A_{e}\right), \quad A_{e}=A_{l}+A_{0}-A_{m} .
\end{gathered}
$$

As in the two-dimensional case (part $2, \mathrm{Wu} 1971 b$ ), $\widetilde{C}_{T, 0}$ will be called the 'proportional-loading parameter', $A_{e}$ may be regarded as an 'effective virtual mass' for thrust. The corresponding optimum mean power coefficient is

$$
C_{P}=2\left(\xi_{0} \sigma\right)^{2}(1-\lambda) A_{e}
$$

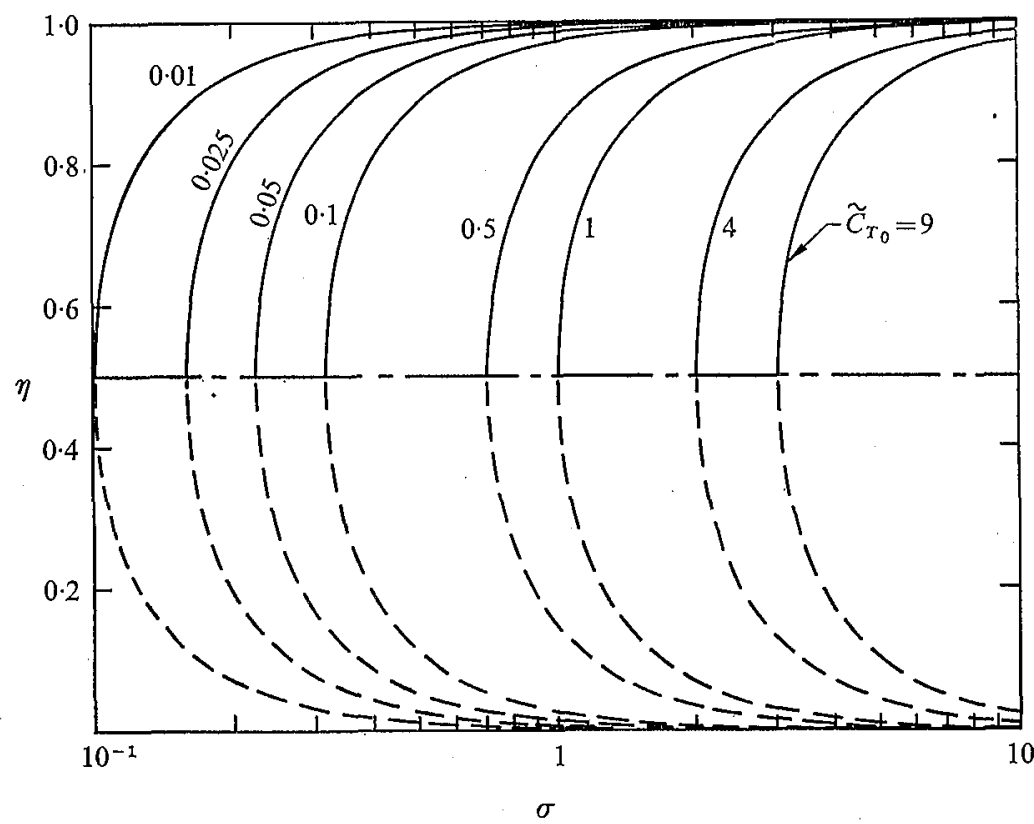

Fradre 5. Variation of the maximum and minimum efficiencies with the reduced frequency $\sigma$ for fixed $\widetilde{C}_{T, 0}$.

The maximum and minimum efficiency, under condition (64), are therefore

$$
\begin{aligned}
\eta_{\max } & =\frac{1}{2}\left(1+\lambda_{1}\right)=\frac{1}{2}\left[1+\left(1-\widetilde{C}_{T, 0} / \sigma^{2}\right)^{\frac{1}{2}}\right], \\
\eta_{\min } & =1-\eta_{\max } .
\end{aligned}
$$

It is of interest to observe that the above optimum efficiency of a slender fish behaves very much like the two-dimensional solution obtained in part 2 (Wu 1971b). The optimum solution depends again on two parameters, $\sigma$ and $\widetilde{C}_{T, 0}$, and is real only for $\sigma \geqslant \sigma_{c}$. As $\sigma$ increases from $\sigma_{c}, \eta_{\max }$ increases towards unity, whereas $\eta_{\min }$ diminishes to zero, both rather rapidly from $\eta=0.5$, as shown in figure 5. For $\sigma \gg \sigma_{c}$,

$$
\eta_{\max } \simeq 1-\tilde{C}_{T, 0} /(2 \sigma)^{2}
$$


Another quantity of importance is the phase velocity $c$ of body wave form relative to the swimming speed $U$. For the optimum shape (73), the phase velocity is $c=\omega / \lambda \sigma=U / \lambda$, or

$$
U / c=\left(1-\tilde{C}_{T, 0} / \sigma^{2}\right)^{\frac{1}{2}}
$$

For $\sigma \gg \sigma_{c}, U$ is therefore only very slightly less than $c$.

To estimate the order of magnitude of $\sigma_{c}$, we equate the thrust $T$ and the frictional drag, assuming no flow separation, so that

$$
D=\frac{1}{2} \rho U^{2} C_{D} S_{f}=T=\frac{1}{4} \rho U^{2} l_{m}^{2} C_{T, 0}
$$

where $C_{D}$ is the skin-frictional drag coefficient based on the total wetted surface $S_{f}$. This leads to

$$
\widetilde{C}_{T, 0}=\left(\frac{C_{D}}{\xi_{0}^{2}}\right)\left(\frac{2 S_{f}}{A_{0}-A_{m}+A_{l}}\right)
$$

the factor with $S_{f}$ being of order $O\left(\delta^{-1}\right)$ for small slenderness parameter $\delta$. This indicates that, if the comparison is based on the same $\left(C_{D} / \xi_{0}^{2}\right)$, then $\sigma_{c}$ for slender fish would be somewhat greater than its counterpart of a two-dimensional flexible plate. This may suggest that, at the same degree of high efficiency, a slender fish would be more likely employing a higher reduced frequency motion than a two-dimensional flapping wing based on the same $\left(C_{D} / \xi_{0}^{2}\right)$. The result of nearly uniform amplitude of transverse motion along the slender fish from the maximum span to tail is another important feature of the solution. With no such requirement for the front part of body, it seems that motions with somewhat reduced amplitude in the front part help keep the recoil small.

\section{The vortex wake of optimum motion}

It is instructive to investigate the vortex wake generated by the optimum body motion (73). The corresponding transverse flow velocity is

$$
V(x, t)=D h=i \xi_{0} \omega(1-\lambda) \exp [i(\omega t-\lambda \sigma x)] \quad\left(x, y \in S_{b}\right)
$$

so that, with $\tau_{1}=t+\left(x_{1}-x\right) / U$,

$$
V\left(x_{1}, \tau_{1}\right)=i U \xi_{0} \sigma(1-\lambda) \exp \left[-i \sigma(x-U t)+i \sigma(1-\lambda) x_{1}\right] .
$$

Substituting this retarded value of $V$ in (31), we obtain, for $0<x<1,|y|<b_{0}$, $\Gamma=2 i U \xi_{0} \sigma(1-\lambda) \exp [-i \sigma(x-U t)]\left\{\left(b_{0}^{2}-y^{2}\right)^{\frac{1}{2}}+i \sigma(1-\lambda)\right.$

$$
\left.\times \int_{0}^{x_{*}}\left(b_{1}^{2}-y^{2}\right)^{\frac{1}{2}} \exp \left[i \sigma(1-\lambda) x_{1}\right] d x_{1}\right\}
$$

For the maximum efficiency, we take in the above equation $\lambda=\lambda_{1}$, which makes $\left(1-\lambda_{1}\right) \ll 1$ for $\sigma \gg \sigma_{c}$ (see (74)). Therefore the second term inside the curly bracket can be neglected, and, by (40), the vorticity $\gamma_{2}$ of the wake is

$$
\gamma_{2}(x, y, t)=-2 U \xi_{0} \sigma^{2}\left(1-\lambda_{1}\right) \exp [-i \sigma(x-U t)]\left\{\left(b_{0}^{2}-y^{2}\right)^{\frac{1}{2}}+O\left(\sigma\left(1-\lambda_{1}\right)\right)\right\}
$$


valid for $(x, y) \in S_{w}, 0<x<1 . \gamma_{2}$ has an elliptical spanwise distribution, and its magnitude is approximately proportional to $C_{T, 0} / \xi_{0}$. From the above solution some of the important features of the vortex wake can be demonstrated.

For a fixed station $x$, write the phase angle of the transverse motion $h$ as $\theta=\omega t-\lambda_{1} \sigma x$ so that $\theta$ increases by $2 \pi$ as $t$ varies over a period $2 \pi / \omega$. Taking the real part of (73) and (80) for physical interpretation, we obtain the qualitative picture of $h(x, t)$ and $\gamma_{2}(x, 0, t)$ as shown in figure 6 . As $\gamma_{2}$ leads $h$ in phase by $\pi-\left(1-\lambda_{1}\right) \sigma x$, they are nearly opposite in phase, since the phase angle $\Delta \theta=\left(1-\lambda_{1}\right) \sigma x$ is small, but increases distally. In a reference frame fixed
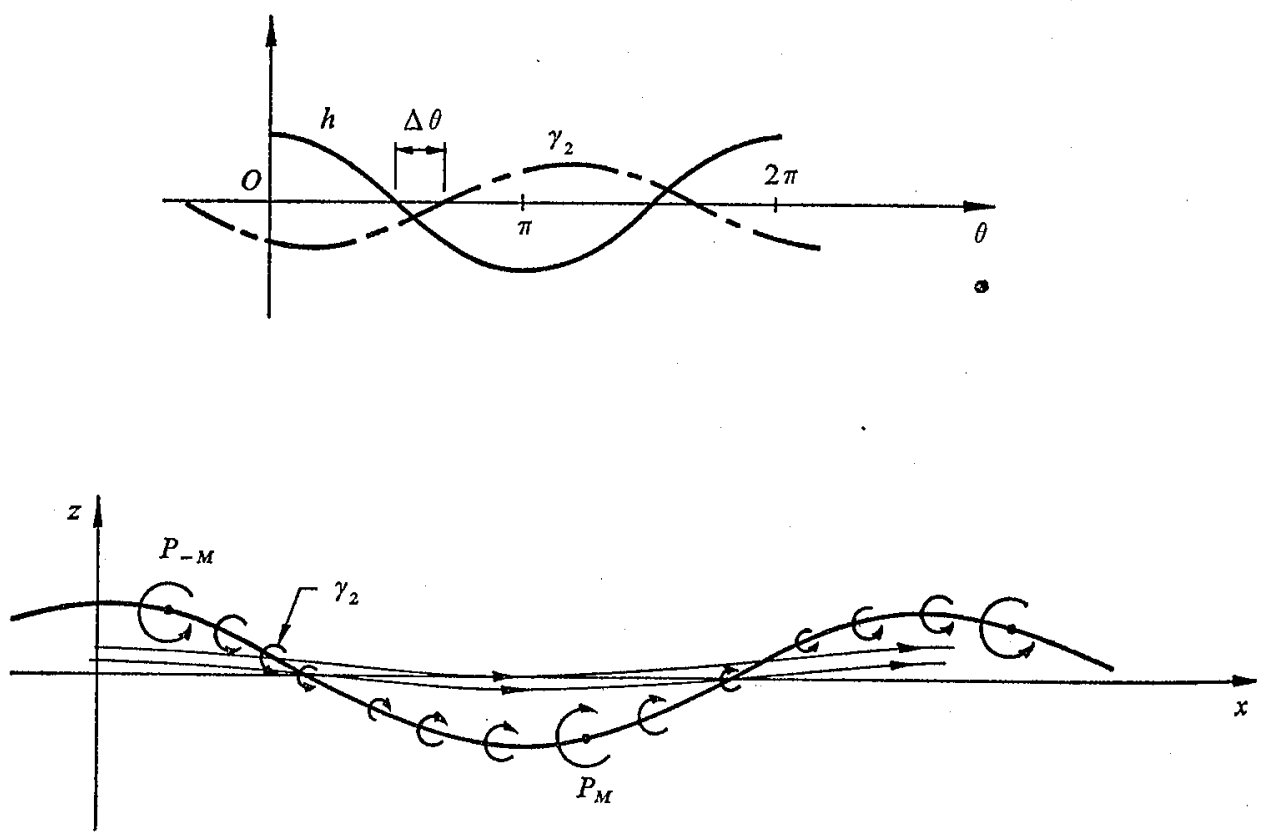

Figure 6. The vortex walke of the optimum movement. $\gamma_{2}$ reaches the maximum at $P_{M}$ and minimum at $P_{-M}$.

relative to the undisturbed fluid, the section $x$ will traverse a sinusoidal trajectory, shedding in the course of time a trail of vortices $\gamma_{2}$ as shown qualitatively in figure 6 . The velocity of the fluid particles along the $x$-axis induced by this system of $\gamma_{2}$ vortex sheet is seen clearly to move backwards from the body, resulting in a 'jet stream', to which the forward thrust $T$, regarded as the reaction to this jet flow momentum generated at this optimum operational state, can be wholly attributed. When the viscous wake produced by the diffusion of the vorticity left behind from the boundary-layer flow is considered in addition, the entrainment of fluid due to the viscous wake is opposite in direction to that caused by $\gamma_{2}$, thus leaving no net flow momentum at large distances behind the body, if it is self-propelling. This explains the basic mechanism of swimming of a slender fish. 
I am very much indebted to Professor M. J. Lighthill for his interest in this work, and particularly for his kindness in giving me the privilege of knowing his great work (1970) prior to its publication. The enlightening discussions with him have enabled me to draw instructive comparisons between the conclusions reached in his work and in this part of my own. I am also grateful to Professor T. K. Caughey for some general discussions. This research was partially sponsored by the National Science Foundation, under Grant GK 10216, and by the Office of Naval Research, under Contract N00014-67-A0094-0012.

\section{Appendix}

We discuss here the miscellaneous effects upon the swimming performance due to geometrical asymmetry, finite body thickness, and unsteady forward speed.

\section{(i) Effect of geometrical asymmetry}

The geometrical asymmetry referred to here is the two side edges $y=-b_{1}(x)$ and $y=b_{2}(x)$ being not necessarily symmetric in $y$, i.e. $b_{1} \neq b_{2}$. The problem of the cross-flow in the front part with leading side edges $\left(-l_{n}<x<0\right)$ is again classical. The complex velocity $\Omega$ is

$$
\Omega=\frac{d f}{d \zeta}=i V(x, t)\left\{\left(\zeta+\frac{b_{1}-b_{2}}{2}\right)\left[\left(\zeta+b_{1}\right)\left(\zeta-b_{2}\right)\right]^{-\frac{1}{2}}-1\right\} \quad\left(-l_{n}<x<0\right),
$$

which can be obtained simply by introducing an appropriate translation of the co-ordinates. The complex potential is given by integration,

$$
f=i V\left\{\left[\left(\zeta+b_{1}\right)\left(\zeta-b_{2}\right)\right]^{\frac{1}{2}}-\left(\zeta+\frac{b_{1}-b_{2}}{2}\right)\right\} \quad\left(-l_{n}<x<0\right),
$$

from which the complex acceleration potential follows simply as $F=D f$. The constant of integration in (A 2) has been so chosen as to have the pressure vanish at infinity.

In the segment posterior to the maximum span section, the solution of the acceleration potential $F$ can be written, in view of the analogy between $F$ and $f$ given in (17) and (18) and here applied to (A 2), as

$$
F=(i D V)\left\{\left[\left(\zeta+b_{1}\right)\left(\zeta-b_{2}\right)\right]^{\frac{1}{2}}-\left(\zeta+\frac{b_{1}-b_{2}}{2}\right)\right\} \quad(x>0) .
$$

Using the above solution of $f$ and $F$ in (41), one readily verifies that the lift per unit length, $\mathscr{L}(x, t)$, is given by the same expression $(41 a, b)$ with

$$
A(x)=\pi\left[\frac{1}{2}\left(b_{1}+b_{2}\right)\right]^{2} \text {. }
$$

This result shows that a small asymmetry does not affect the integrated transverse force in this slender body approximation, as should be expected.

\section{(ii) Effect of finite body thickness}

We next relax the restriction of zero body thickness by considering the class of slender bodies whose transverse cross-sections can have arbitrary shape, except that the rear part of body posterior to the maximum span section still 
has pointed, or even cusped, side edges, a principal feature retained here to represent approximately the elongated spiny caudal, dorsal and ventral fins of various kinds of fishes. In addition, the body shape, when at rest, is assumed to be symmetric in both $y$ and $z$. The analysis originally advanced by Lighthill $(1960 a)$ suffices to assert that the transverse lift per unit length, $\mathscr{L}(x, t)$, as given by $(41 a)$ still holds valid for arbitrary cross-sectional shape in the front part anterior to the maximum span section provided the virtual mass $\rho A(x)$ is referred properly to the prescribed shape.

As for the rear part of body containing the sharp trailing side edges, the method of conformal mapping can be utilized to determine the cross-flow. Suppose that the shape of body cross-section in the $\zeta=y+i z$ plane at station $x$, with span $-b(x)<y<b(x)$ and given thickness $z= \pm g(y ; x)$, is mapped conformally onto the slit $-\beta(x)<\operatorname{Re} \xi<\beta(x)$ in the complex $\xi$-plane by

$$
\zeta=\zeta(\xi) \text { or } \xi=\xi(\zeta), \text { with } \beta(x)=\xi(b(x)),
$$

where $\zeta(\xi)$ is analytic function of $\xi$, satisfying the condition $d \zeta \mid d \xi \rightarrow 1$ as $|\zeta| \rightarrow \infty$. It is convenient to keep the complex acceleration potential $F$ invariant under transformation (A 4), i.e. $F(\zeta)=F(\zeta(\xi)$ ). The boundary conditions for the problem $F$ in the body frame of reference are

$$
\begin{aligned}
& \Psi^{ \pm}=\operatorname{Im} F^{ \pm}=0 \quad(\operatorname{Im} \xi= \pm 0, \quad-\beta(x)<\operatorname{Re} \xi<\beta(x)), \\
& \Phi=\operatorname{Re} F=0 \quad(\operatorname{Im} \xi=0, \quad|\operatorname{Re} \xi| \geqslant \beta(x)), \\
& F \rightarrow(i D V) \xi \quad(|\xi| \rightarrow \infty) \text {. }
\end{aligned}
$$

Condition (A 5) follows from the fact that body surface is a streamline, $\psi=0$, and hence $\Psi=D \psi=0$. Condition (A 6 ) holds valid both on the trailing vortex sheet (on which $D \phi=0$ ) and outside the vortex sheet (where $\phi=0$ ), including the Kutta condition at $\operatorname{Re} \xi= \pm \beta(x)$. Condition (A 7) follows from the velocity condition $w \rightarrow i V$, and hence $f \rightarrow i V \zeta$ as $|\zeta| \rightarrow \infty$ in the physical $\zeta$-plane. The only bounded solution of $F$ satisfying these conditions is clearly

$$
F_{\text {b.P. }}=(i D V)\left(\xi^{2}-\beta^{2}\right)^{\frac{1}{2}}
$$

where the subscript b.f. denotes the solution in the body frame. The solution of $F$ in the physical $\zeta$-plane in the reference frame fixed with respect to the fluid at infinity is readily obtained by superposing a uniform velocity $w_{0}=-i V$, corresponding to adding $F_{0}=-i(D V) \zeta$ to $F_{\mathrm{b} . \mathrm{f} .}$, giving

$$
F=(i D V)\left\{\left[\xi^{2}(\zeta)-\beta^{2}(x)\right]^{\frac{1}{2}}-\zeta\right\}
$$

which falls off at infinity and is bounded at the trailing side edges $\zeta= \pm b$.

The corresponding pressure at the body surface is given by

$$
p^{ \pm}-p_{\infty}=-\rho \Phi \pm= \pm \rho(D V)\left\{\left[\beta^{2}-\xi^{2}\right]^{\frac{1}{2}}-z(\xi)\right\} \quad(\xi \text { real, } \quad|\xi|<\beta),
$$

in which the $+($ or - ) sign is for the upper (or lower) half of body, and

$$
z(\xi)=g(y(\xi))
$$


is the thickness function. The lift per unit length is therefore

$$
\begin{aligned}
& \qquad \mathscr{L}(x, t)=\oint_{\mathscr{A}} p d y=\oint_{\mathscr{C}} p(\xi) \frac{\partial y}{d \xi} d \xi=-\rho[D V(x, t)] A(x) \quad(0<x<1) \\
& \qquad A(x)=\int_{-\beta}^{\beta}\left[\left(\beta^{2}-\xi^{2}\right)^{\frac{1}{2}}-z(\xi)\right] \frac{\partial y}{\partial \xi} d \xi
\end{aligned}
$$

The above $\mathscr{L}(x, t)$ has the same expression as $(41 b)$ for the case of planar body, but now the virtual mass $\rho A(x)$ includes the effect of body thickness.

\section{(iii) Time-dependent forward speed}

The foregoing discussion based on constant forward speed can be easily extended to the general case of time-dependent rectilinear velocity $U(t)$, the motion being now assumed to start from $t=0$. If the velocity is again linearized with respect to the instantaneous free-stream velocity $U(t)$, the formulation of the problem of $f$ in the front portion $\left(-l_{n}<x<0\right)$ and of $F$ in the rear portion $(0<x<1)$ remain unchanged in form. Consequently the solution of $F$ given by (22) and (24) still holds valid even when $U$ depends on $t$. Only when $f$ is calculated by integrating (15) does the effect of time dependence of $U$ arise. For this purpose, we introduce, as was done previously in part 1 (1971 $a$ ) for the twodimensional case, the new variable

$$
s(t)=\int_{0}^{t} U(t) d t
$$

assuming its inverse function $t=t(s)$ is unique so that $U=U(t(s))=\tilde{U}(s)$ is a one-valued function of $s$. Equation (15) then becomes

$$
\left(\frac{\partial}{\partial s}+\frac{\partial}{\partial x}\right) f=\frac{1}{U} F
$$

Regarding $f$ and $F$ as functions of $(x, s)$, we have

$$
f(\zeta ; x, s)=f\left(\zeta ; x_{0}, s+x_{0}-x\right)+\int_{x_{0}}^{x}\left[F\left(\zeta ; x_{1}, s+x_{1}-x\right) / \widetilde{U}\left(s+x_{1}-x\right)\right] d x_{1} .
$$

By making use of this integration formula, we see that the only changes which are needed to modify the previous solution for the present case are the replacement of $V\left(x_{1}, \tau\left(x_{1} ; x, t\right)\right)$ in (26), (28), (31), (32) by $\tilde{V}\left(x_{1}, s+x_{1}-x\right)$, where

$$
V(x, t(s)) \equiv \tilde{V}(x, s) .
$$

The instantaneous thrust $T(t)$, power $P(t)$ and energy loss $E(t)$ are again given by (46), (48) and (51).

\section{REFERENCES}

BAINBRIDGE, R. 1963 J. Exp. Biol. 40, 23.

Cotrant, R. \& HILBmRT, D. 1953 Methods of Mathematical Physics (vol. 1). Interscience. GRAY, J. 1968 Animal Locomotion. London: Weidenfeld \& Nicolson.

HERTEL, H. 1963 Structure-Form-Movement. Mainz: Krausskopf. (English trans. 1966; New York: Reinhold.) 
LAMB, H. 1932 Hydrodynamics (6th edn.). Cambridge University Press.

LighthILL, M. J. 1960 a J. Fluid Mech. 9, 305.

LighthILL, M. J. $1960 b$ J. Roy. Aero. Soc. 64, 373.

LigntнmL, M. J. 1969 Ann. Rev. Fluid Mech. 1, 413.

LigнтнтाL, M. J. 1970 J. Fluid Mech. 44, 265.

MUSKHelishviti, N. I. 1953 Singular Integral Equations. Groningen, Holland: Noordhoff.

Rosen, M. W. 1959 Naval Ord. Test Station Publ. TP 2298.

Walters, V. \& Fiersteine, H. L. 1964 Nature, Lond. 202, 208.

WEYL, H. 1910 Math. Ann. 68, 220.

Wu, T. Y. 1971 a J. Fluid Mech. 46, 337.

Wo, T. Y. $1971 b$ J. Fluid Mech. 46, 521. 